\title{
A six degree-of-freedom fused silica seismometer: Design and tests of a metal prototype
}

\author{
Amit Singh Ubhi, Jiri Smetana, Teng Zhang, Sam Cooper, Leo \\ Prokhorov, John Bryant, David Hoyland, Haixing Miao, and Denis \\ Martynov \\ Institute for Gravitational Wave Astronomy, School of Physics and Astronomy, University of \\ Birmingham, Birmingham B15 2TT, United Kingdom
}

\begin{abstract}
Ground vibrations couple to the longitudinal and angular motion of the aLIGO test masses and limit the observatory sensitivity below $30 \mathrm{~Hz}$. Novel inertial sensors have the potential to improve the aLIGO sensitivity in this band and simplify the lock acquisition of the detectors. In this paper, we experimentally study a compact 6D seismometer that consists of a mass suspended by a single wire. The position of the mass is interferometrically read out relative to the platform that supports the seismometer. We present the experimental results, discuss limitations of our metallic prototype, and show that a compact 6D seismometer made out of fused silica and suspended with a fused silica fibre has the potential to improve the aLIGO low frequency noise.
\end{abstract}




\section{Introduction}

The network of the Advanced LIGO [1] and Advanced Virgo [2] gravitational-wave (GW) detectors observed tens of signals from compact objects with masses up to $150 M_{\odot}[3,4,5$, $6,7]$. Most of the astrophysical sources were categorised as neutron stars and stellar mass black holes, with one event producing an intermediate-mass black hole [7]. The signals were mostly accumulated at frequencies from $30 \mathrm{~Hz}$ up to $500 \mathrm{~Hz}$ due to the diminished response of the detectors at lower and higher frequencies. Despite the sophistication and successful operation of aLIGO's seismic isolation platforms [8, 9], the sensitivity degrades below $30 \mathrm{~Hz}$ due to non-stationary control noise [10, 11, 12].

Improvements of the aLIGO inertial isolation will result in a number of benefits (i-v) for the detectors. Enhanced sensitivity below $30 \mathrm{~Hz}$ will lead to (i) routine observations of intermediate-mass black-holes since heavier compact objects merge at lower frequencies. Observing GWs at lower frequencies also increases the measurement time of mergers [10] and provides (ii) an improved localisation of the sources and enables early-warning alerts to search for the electromagnetic counterparts of events like GW170817 [6]. Furthermore, since the aLIGO lock acquisition process is a complex procedure that involves stabilisation of five longitudinal and tens of angular degrees of freedom [13, 14], suppression of the test mass motion will (iii) simplify the lock acquisition process. The coincident duty cycle of the aLIGO Livingston and Hanford detectors has the potential to (iv) increase from the current $62.2 \%$ [11] up to $\approx 75 \%$ once seismic distortions are further suppressed. Finally, reduced motion of the aLIGO test masses will allow us to increase the beam size in the arm cavities and (v) improve the coating thermal noise $[15,16]$ that limits the aLIGO sensitivity in its most sensitive frequency band around $100 \mathrm{~Hz}[11,17]$.

A number of inertial sensors has been proposed in the literature [18, 19, 20, 21, 22, 23, 24] that have the potential to reduce the aLIGO platform motion and enhance the detector sensitivity. The key problem comes from the coupling of the aLIGO platform tilt to its horizontal motion. This coupling scales very unfavourably as $g / \omega^{2}$, where $g$ is the local gravitational acceleration, and $\omega$ is the angular frequency of the platform motion [25, 26, 27 , 28]. The aLIGO detectors already utilise custom beam rotation sensors [18] developed by the University of Washington to measure the tilt motion of the ground. The next logical step would be to develop new custom sensors for the aLIGO platforms to further suppress their motion.

In this paper, we experimentally investigate a compact $6 \mathrm{D}$ seismometer that has the potential to measure the aLIGO platform motion in all six degrees of freedom [19]. The 6D seismometer is similar to drag-free control, which is a technique employed in satellites to keep the spacecraft a constant distance from a free-falling mass [29]. In contrast to the rotation sensors, we avoid mechanical constraints of the inertial mass and allow it to move freely in all degrees of freedom. The mechanical simplicity, however, leads to digital complexity since we need to diagonalise coupled degrees of freedom. In Sec. 2, we discuss experimental results, show how to diagonalise the coupled degrees of freedom, and compare the sensitivity of the 6D seismometer with commercial sensors. In Sec. 3, we propose a vacuum-compatible 


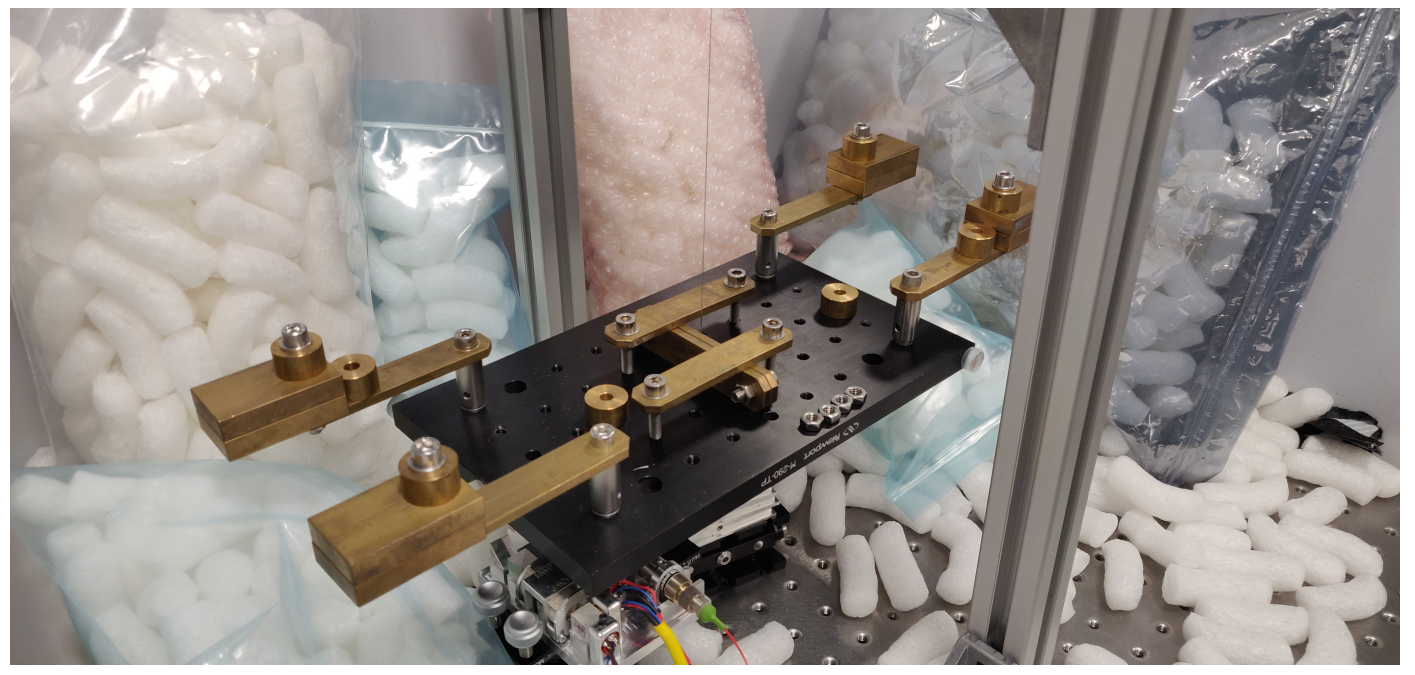

Figure 1. Picture of the isolated metallic prototype with an interferometric sensor.

version of the compact 6D seismometer and discuss the impact for the aLIGO detectors.

\section{Metallic prototype}

In this section, we discuss a metallic prototype, shown in Fig. 1, that was constructed with the aim of understanding the mechanics and dynamics of the system. The purpose was to provide insight for the fused silica design discussed in Sec. 3.

\subsection{Mechanical design}

The prototype used a $21 \mathrm{~cm}$ long, $r=200 \mu \mathrm{m}$ radius stainless steel wire to suspend an aluminium breadboard of dimensions $(254 \times 152.4 \times 12.7) \mathrm{mm}-$ this was used as the base for the proof mass. We added additional brass masses to extend the proof mass and increase its moment of inertia to $I_{\mathrm{RZ}} \approx 0.076 \mathrm{kgm}^{2}, I_{\mathrm{RY}} \approx 0.061 \mathrm{kgm}^{2}, I_{\mathrm{RX}} \approx 0.015 \mathrm{kgm}^{2}$, where RX, RY, and RZ implies rotation around the horizontal $\mathrm{X}$ - and Y-axes, and the vertical Z-axis. With these parameters, we achieved longitudinal eigenmodes along the X-and Y-axes of $1.096 \mathrm{~Hz}$. We set the vertical bounce mode of the suspension at $25 \mathrm{~Hz}$. The rigidity of the vertical mode made the system impermeable to vertical motion of the ground below $10 \mathrm{~Hz}$ and simplified the measurement of the angular motion of the inertial mass. This can be seen in Fig. 4, where above $10 \mathrm{~Hz}$ the vertical commercial L4C seismometer [30] (orange) measures the same as the vertical sensor on the proof mass (red), and below this, the vertical motion is not dominant in the sensor. The torsional (RZ) eigenmode was set at $20 \mathrm{mHz}$.

The tilt ( RX and RY) eigenmodes of the suspended mass depend on the vertical centreof-mass position of the system according to the equation

$$
\omega_{\mathrm{RX}, \mathrm{RY}}^{2}=\left(2 \pi f_{\mathrm{RX}, \mathrm{RY}}\right)^{2} \approx \frac{m g \Delta z+k_{\mathrm{el}}}{I_{\mathrm{RX}, \mathrm{RY}}}
$$

where $\Delta z$ is the centre of mass position relative to the bending point of the bottom part of the wire, $k_{\mathrm{el}}=1 / 2 \sqrt{m g E I_{a}}$ is the elastic restoring coefficient of the wire, $m=3.6 \mathrm{~kg}$ is the 
mass of the seismometer mass, $E=210 \mathrm{GPa}$ is the Young's modulus of the steel wire, and $I_{a}=\pi r^{4} / 4$ is the second area moment of inertia of the wire.

For the configuration used, the tilt modes were $f_{\mathrm{RY}}=84 \mathrm{mHz}$ and $f_{\mathrm{RX}}=167 \mathrm{mHz}$ respectively. We adjusted $\Delta z$ to create a gravitational anti-spring and lower the tilt modes. The centre of mass position was above the bending point by $\approx 0.8 \mathrm{~mm}$, creating an inverted pendulum. The resulting response was such that the effective spring constant $k_{\text {eff }}=m g \Delta z+k_{e l}$ was smaller than the restoring coefficient of the wire, $k_{\mathrm{el}}$. We ensured that the effective spring constant still had a positive value so that the system remained stable. The key problem with reducing $k_{\text {eff }}$ was that balancing the mass became difficult due to the plastic deformation of the steel wire as discussed in Sec. 2.2.

The system had quality factors of 2000 for the horizontal modes and 700 for the tilt modes. These quality factors were limited by air damping, and by mechanical losses in the metal fibres and in the clamping mechanisms. The large quality factors for a steel wire suspension led to significant motion of the inertial mass. We reduced the quality factors down to 70 and 3.5 for the longitudinal and tilt modes with eddy current damping. The effect of damping can be seen in Fig. 3 as the resonant peaks are broad.

\subsection{Plastic deformations}

The key experimental challenge of balancing the softly suspended mass came from plastic deformations in the steel wire. The wire stress due to the wire tension is given by the equation

$$
\sigma_{t}=\frac{m g}{\pi r^{2}}=281 \mathrm{MPa}
$$

The stress, $\sigma_{t}$, was already close to the yield stress of the wire material (350 MPa) during the experiment. In addition to the stress from the tension, $\sigma_{t}$, DC tilts of the mass led to the extra stress near the surface of the wire given by the equation

$$
\sigma_{\mathrm{RY}} \approx k_{\mathrm{el}} \frac{\alpha_{\mathrm{RY}}}{r^{3}}=500 \frac{\alpha_{\mathrm{RY}}}{0.1 \mathrm{rad}} \mathrm{MPa}
$$

where $\alpha_{\mathrm{RY}}$ is the DC misalignment of the suspended mass around the RY axis. The total stress inside the wire has exceeded the yield stress for misalignment angles of only $\approx 10^{-2} \mathrm{rad}$. As a result of the plastic deformation, the equilibrium position of the suspended mass continuously changed during the balancing procedure that consisted of adding small masses to either side of the mass. We found that plastic deformations made the balancing procedure ineffective when $k_{\text {eff }}$ approached zero.

\subsection{Optical readout}

Measurement of the proof mass was accomplished using interferometric readout with two Homodyne Quadrature Interferometers (HoQIs) [31]. We utilised only two sensors since this number is sufficient to understand the key cross-couplings between $\mathrm{X}$ and RY, and between $\mathrm{Y}$ and RX degrees of freedom as discussed in Sec. 2.4. One horizontal HoQI measured the translational motion $(\mathrm{X})$, and another HoQI measured the vertical position at the end of the 


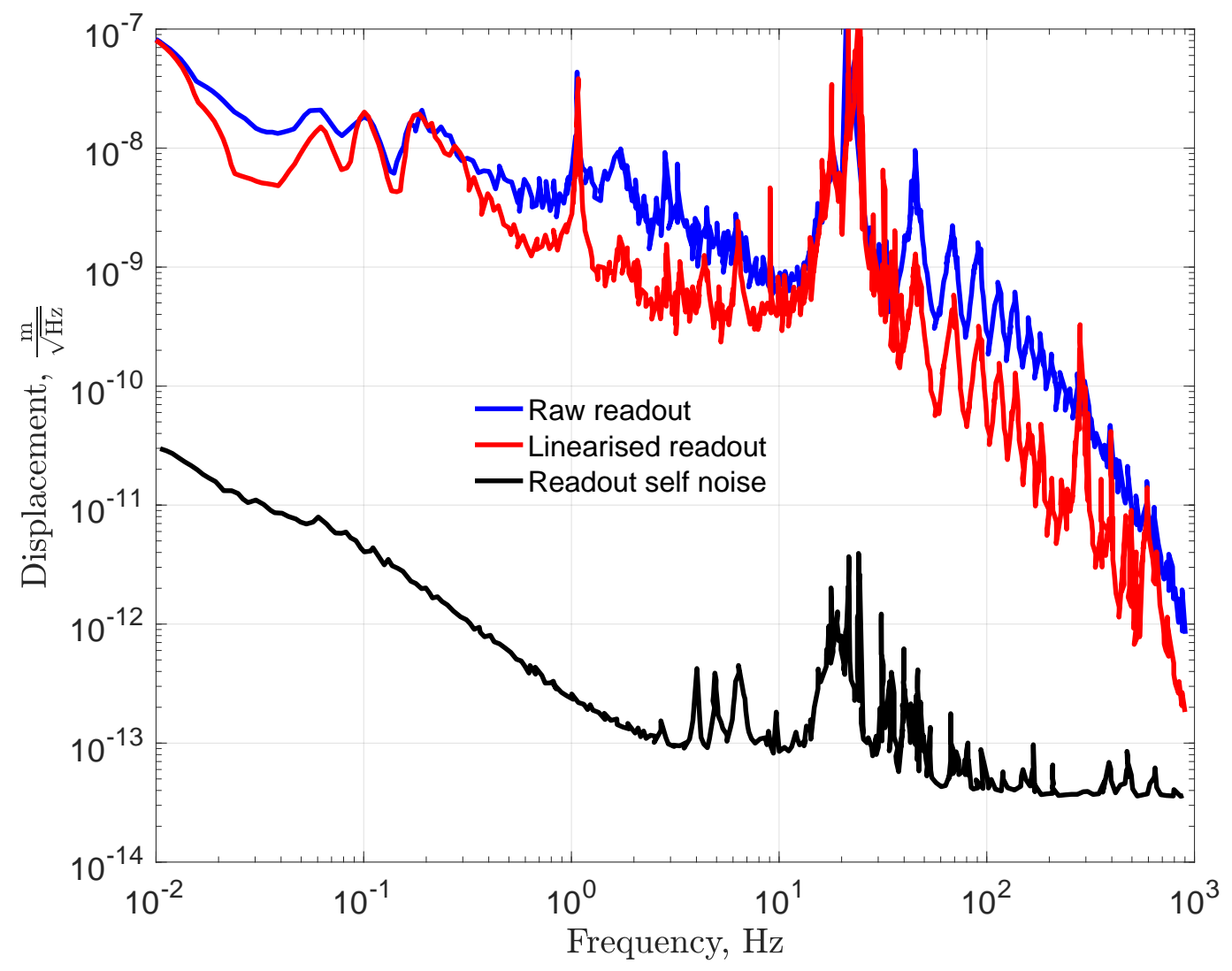

Figure 2. Example of nonlinearities witnessed in the interferometric readout during the experiment. The nonlinearities are strongly suppressed by fitting the Lissajous figures of the compact interferometers with ellipses. The black curve shows the self noise of the sensor measured with stationary mirrors.

proof mass. The vertical motion was directly converted to RY motion of the mass below $10 \mathrm{~Hz}$ since the vertical mode of the suspension was rigid as discussed in Sec. 2.1.

HoQIs are compact Michelson interferometers that measure the position of the test mass relative to the reference mirror. The longitudinal range of the sensors is $>10 \mathrm{~cm}$ since HoQIs measure the position with two polarisations and count optical fringes of the Lissajous figures [31]. The angular working range of the sensors is $1 \mathrm{mrad}$, therefore, isolation from environmental disturbances was necessary to keep the test mass within the HoQI working range as discussed in Sec. 2.5. Ideally, in order to achieve the best performance of the HoQI sensors, the RMS motion of the mass should be less than one fringe of the interferometer to reduce nonlinear effects in the data. However, the linearisation algorithms [31, 32] improve the broadband noise and up-conversion of large motion in the spectra as shown in Fig. 2. The linearisation can be further improved above $30 \mathrm{~Hz}$ by a more accurate ellipse fitting of the Lissajous figures [31, 32]. 


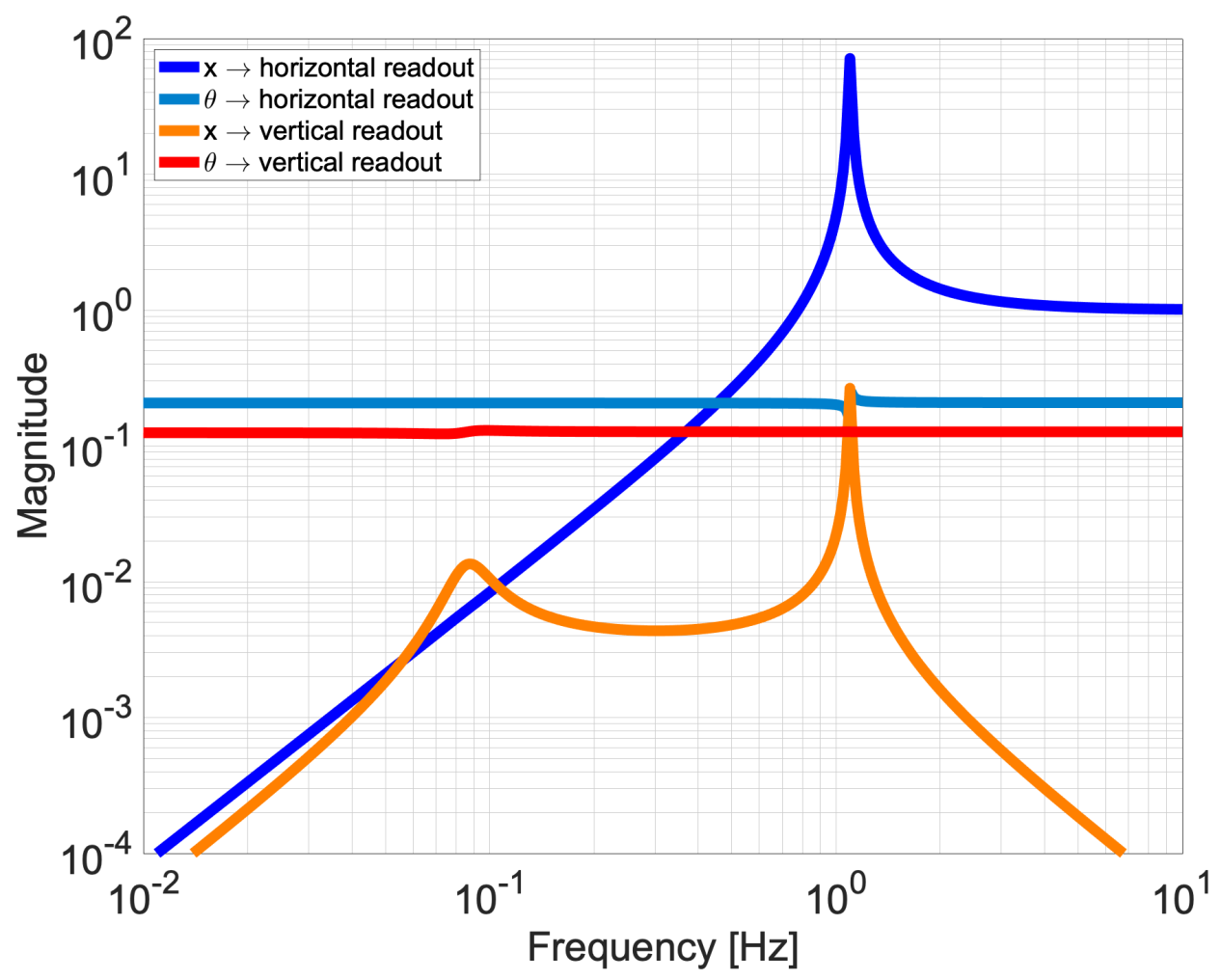

Figure 3. Transfer functions illustrating the coupling between the horizontal and vertical readouts due to the input platform translation $x$, and platform tilt $\theta$.

\subsection{Mechanical cross-couplings}

In the analytical study of the system dynamics [19], we found that the vertical (Z) and torsion (RZ) modes are independent from the other degrees of freedom. For a well aligned and balanced system, the cross-couplings only occur for the $\mathrm{X}$ and RY degrees of freedom and, similarly, for the Y and RX degrees of freedom. Further cross couplings can occur due to sensor misalignment, which will result in a more complex transfer function matrix. In this paper, we neglect second order cross couplings and experimentally investigate $\mathrm{X}$ and $\mathrm{RY}$ frequency-dependent coupling and its diagonalisation.

Fig 3 shows the couplings between the platform motion in X and RY degrees of freedom and readout of the inertial mass position. The platform motion in $\mathrm{X}$ strongly couples to the horizontal readout at and above the suspension resonance in X. Below the resonance, the inertial mass moves in common with the platform and the seismometer response is suppressed. In contrast to the 1D tiltmeters [18], the platform X motion also couples to the RY motion of the mass. Tilt motion of the platform has a minor effect on the mass motion in $\mathrm{X}$ and $\mathrm{RY}$ but moves the local sensors relative to the inertial mass and strongly couples to the readout.

In the case where we infer the $\mathrm{X}$ motion of the platform by looking only at the horizontal readout, we will get the typical tilt-to-horizontal coupling [25, 26, 27, 28]. This coupling is 


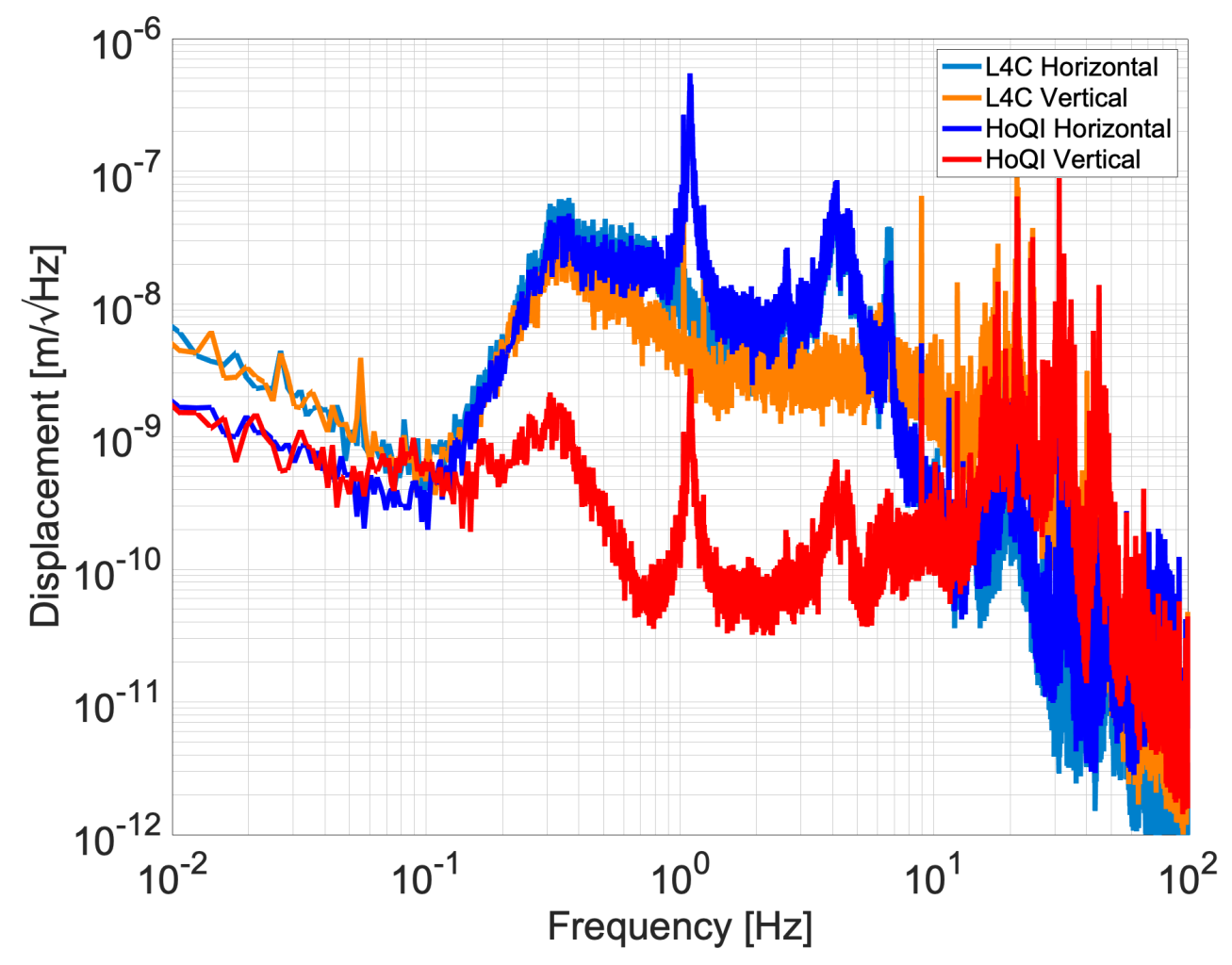

Figure 4. Comparison of measured motion between the compact 6D seismometer and commercial L4C geophones. The translational readout of the seismometer (blue) is similar to the L4C signal (light blue) above $100 \mathrm{mHz}$. The vertical readout (red) measures tilt motion of the inertial mass up to $10 \mathrm{~Hz}$ and vertical motion of the platform above this frequency. The vertical L4C (orange) measures the same motion as the vertical HoQI above the bounce mode resonance at $25 \mathrm{~Hz}$.

present in all 1D seismometers and is given by the equation

$$
\hat{x}=x-\frac{g}{\omega^{2}} \theta,
$$

where $x$ is the translational platform motion, $\theta$ is its RY motion, and $\hat{x}$ is the inferred motion of the platform. In order to avoid the coupling given by Eq.(4), we infer the horizontal motion of the platform from both the horizontal and angular positions of the test mass according to the equation

$$
\left(\begin{array}{l}
\hat{x} \\
\hat{\theta}
\end{array}\right)=\left(\begin{array}{ll}
T_{\mathrm{x} \rightarrow \mathrm{h}} & T_{\theta \rightarrow \mathrm{h}} \\
T_{\mathrm{x} \rightarrow \mathrm{v}} & T_{\theta \rightarrow \mathrm{v}}
\end{array}\right)^{-1}\left(\begin{array}{l}
x_{m} \\
z_{m}
\end{array}\right),
$$

where $x_{m}$ and $z_{m}$ are horizontal and vertical measurements of the HoQI sensors as discussed in Sec. 2.3 and the frequency dependent transfer matrix $T$ is analytically derived and shown in Fig. 3.

\subsection{Sensitivity}

In the laboratory conditions, apart from ground motion, the soft suspension is susceptible to sources of environmental noise, such as air pressure and density fluctuations. The fluctuations 


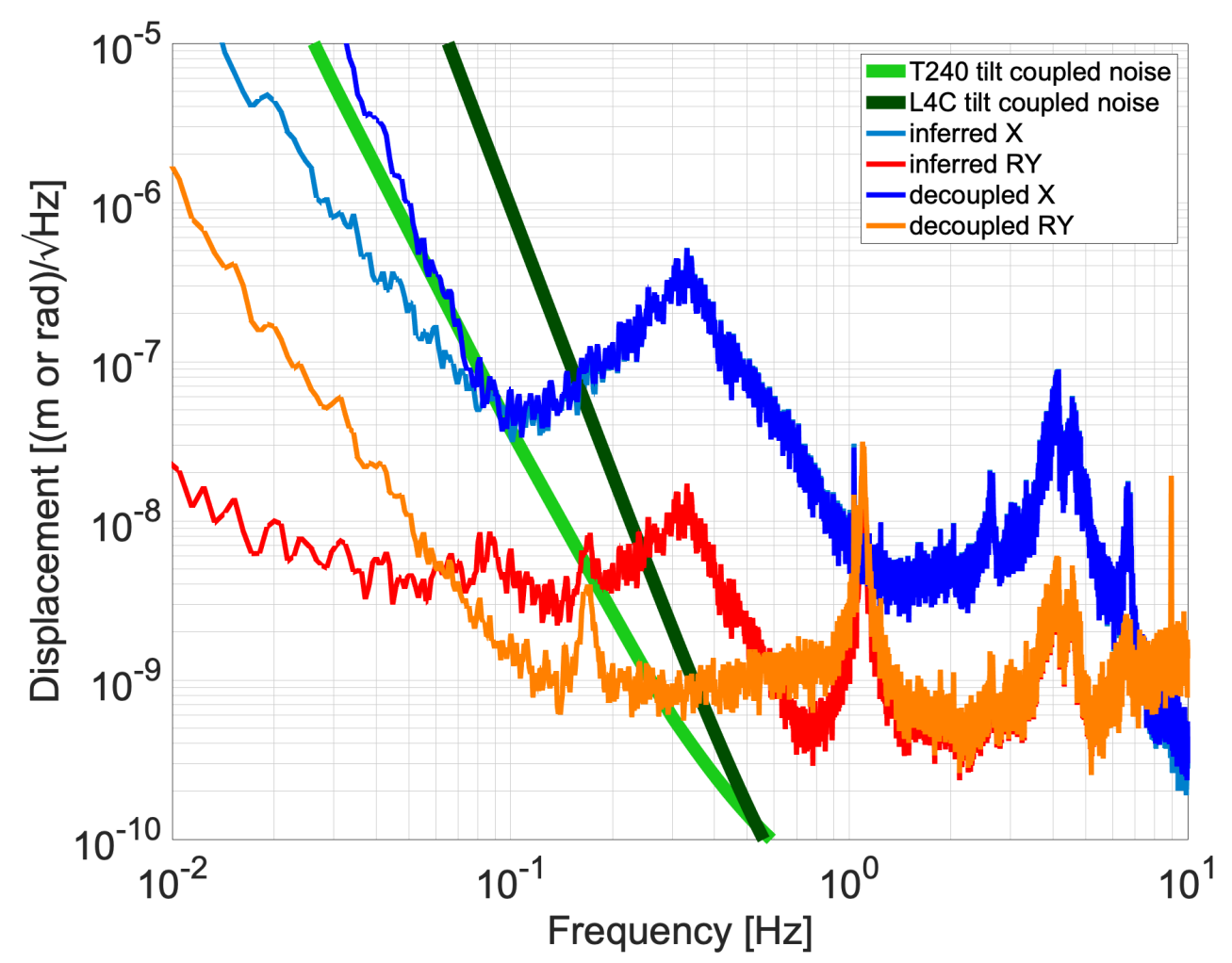

Figure 5. The figure shows the difference between the plant inversions of the translational and tilt modes with and without taking into account the $\mathrm{X}$ and RY cross-couplings. The orange curve shows the translation motion decoupled from the tilt mode such that the microseismic motion is removed. From the current sensitivity of the prototype, we see that the decoupled translational motion has the same noise floor as the tilt coupled T240 noise (green), and has better sensitivity than the L4C (dark green).

move the test mass and couple to the position readout of the mass. To minimise these effects, we placed the system inside a foam padded box that acted as a shield from the local air currents. The box improved the environmental noise by a factor of 30 at $10 \mathrm{mHz}$. A further reduction by of factor 100 was achieved by back filling the box with foam pellets seen in Fig. 1. Despite this reduction of environmental noises, our tilt measurement was still limited by air currents below $100 \mathrm{mHz}$. We expect that environmental noises will disappear once we install the seismometer in vacuum.

Apart from the environmental noise sources, our readout signals measured the platform motion since the interferometric readout noise and thermal noises were significantly smaller compared to the ground vibrations in our experiment. We compared the readouts of our seismometer with commercial L4C sensors [30] as shown in Fig. 4. The L4Cs were colocated and co-aligned with the 6D seismometer to maximise coherence between the sensors. Fig. 4 shows the comparison between measured signals before plant inversion. The spectra for the HoQI horizontal and L4C horizontal are comparable because the resonances for both devices are the same $(\approx 1 \mathrm{~Hz})$. The $\mathrm{Q}$ factor for our seismometer is larger than that of the L4C hence Fig. 4 shows the more prominent $1 \mathrm{~Hz}$ resonance in the HoQI spectra. From these 
measurements we find that the compact 6D noise floor in air is already better than that of the L4Cs below $100 \mathrm{mHz}$.

According to our estimation of the platform translational and tilt motion, the horizontal HoQI sensor measures tilt motion below $100 \mathrm{mHz}$ and translational motion at the other frequencies. The vertical HoQI sensor measures tilt and inertial mass motion due to environmental noise below the tilt resonance at $85 \mathrm{mHz}$. Translational motion couples to the vertical tilt readout between the tilt and translational resonances of the suspension according to the transfer functions shown in Fig. 3. At frequencies from $1.5 \mathrm{~Hz}$ to $10 \mathrm{~Hz}$, the vertical sensor measures the platform tilt motion, and vertical platform motion couples to the readout at higher frequencies.

We have diagonalised our readout signals according to Eq. (5) as shown in Fig. 5. The tilt-decoupled horizontal motion $\hat{x}$ is larger than the inferred horizontal motion without tilt subtraction since our tilt measurement is limited by the environmental noises below $100 \mathrm{mHz}$. However, the achieved sensitivity is comparable to the tilt-decoupled sensitivity of the Trillium T240 sensors [33] that are currently installed in the aLIGO detectors. We calculated the tilt-decoupled power spectrum of the T240 sensor noise according to the equation

$$
S_{\mathrm{T} 240}^{x}=S_{\mathrm{T} 240}\left(1+\frac{2 g^{2}}{\omega^{4} \Delta L^{2}}\right),
$$

where $\Delta L=1 \mathrm{~m}$ is the separation between two vertical T240 seismometers used for the measurement of the platform tilt motion, $S_{\text {T240 }}$ is the power spectrum of the T240 own noise, $g / \omega^{2}$ is the tilt-to-horizontal coupling coefficient, and a factor of 2 in the brackets accounts for two T240 seismometers utilised in the tilt measurement.

Fig. 5 also shows the decoupling of the horizontal platform motion from the tilt measurement at frequencies between $85 \mathrm{mHz}$ and $1 \mathrm{~Hz}$. Apart from the platform tilt motion, we can also see coupling from the other degrees of freedom in $\hat{\theta}$. The resonance at $167 \mathrm{mHz}$ corresponds to the RX eigen mode and the peak at $1.09 \mathrm{~Hz}$ corresponds to the coupling of the $\mathrm{Y}$ motion. We conclude that our sensors were not ideally aligned to measure only $\mathrm{X}$ and $\mathrm{RY}$ degrees of freedom. We leave this coupling problem for further experimental investigations.

\section{Fused silica seismometer}

From the lessons learned in Sec. 2, we propose to build the seismometer for GW detectors from fused silica and suspend the mass using a fused silica fibre, similar to the aLIGO test masses [34]. The design is compact $(\approx 0.5 \mathrm{~m}$ scale $)$ compared to the larger $(\approx 1.2 \mathrm{~m})$ scale $6 \mathrm{D}$ seismometer [19] that the authors investigated in collaboration with VU, Amsterdam. The compact design allows us to (i) achieve low drift rates of the suspended mass as discussed in Sec. 3.1, (ii) utilise commercially available fused silica masses, and (iii) remove the angular control noise from the aLIGO sensitivity band.

We choose fused silica for the design of the compact 6D seismometer due to its low thermal expansion coefficient and low mechanical dissipation. In this section, we consider two designs of the seismometer with the tilt eigenmode at $50 \mathrm{mHz}$ and $100 \mathrm{mHz}$, find the own 


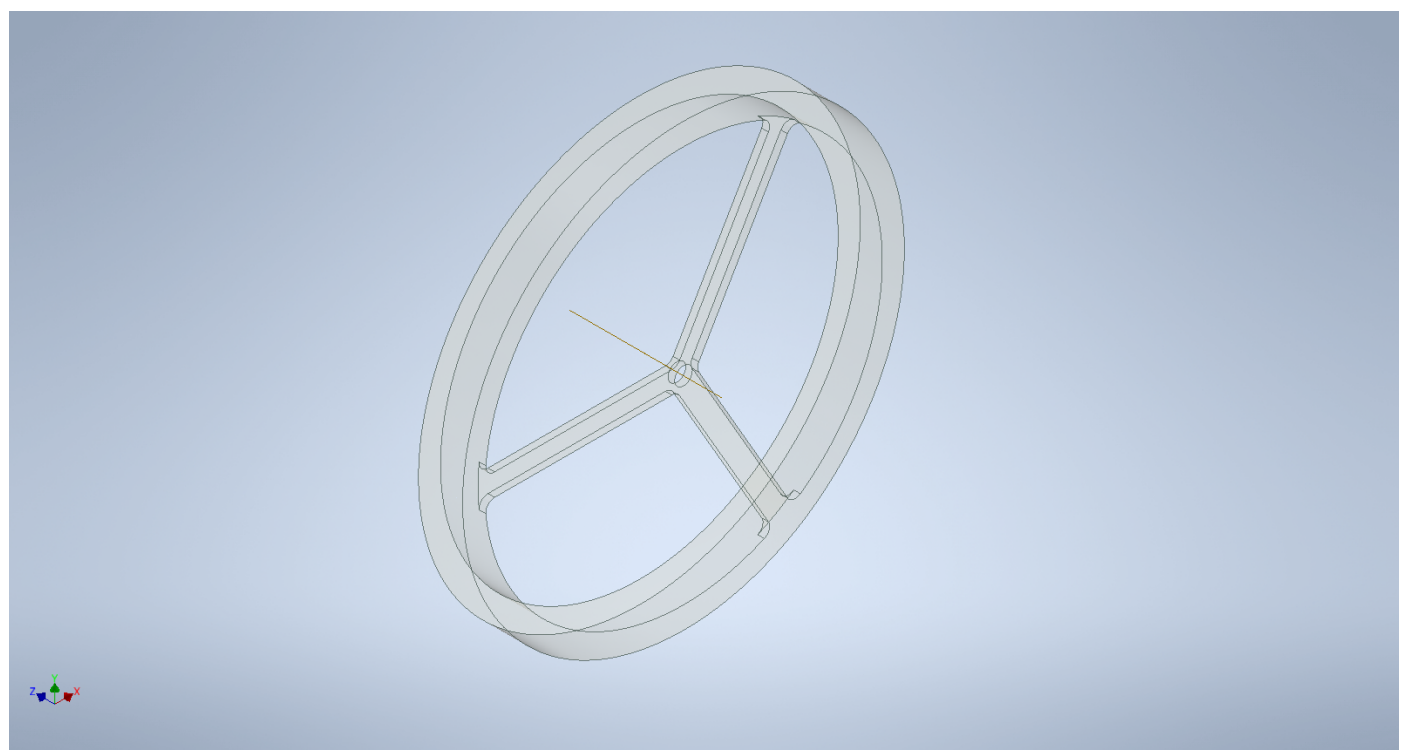

Figure 6. Model of the proposed fused silica compact 6D seismometer for the aLIGO active isolation platforms.

Table 1. A list of parameters and nominal values

\begin{tabular}{ccc}
\hline \hline Parameters & Description & Value \\
\hline$m$ & Mass & $3.25 \mathrm{~kg}$ \\
\hline$R$ & Mass radius & $0.25 \mathrm{~m}$ \\
\hline$L$ & Wire length & $0.4 \mathrm{~m}$ \\
\hline$r$ & Fibre radius at bending points & $120 \mu \mathrm{m}$ \\
\hline$f_{\mathrm{X}, \mathrm{Y}}$ & Translational resonance & $0.8 \mathrm{~Hz}$ \\
\hline$f_{\mathrm{RX}, \mathrm{RY}}$ & Tilt resonance & $50 \mathrm{mHz}$ or $100 \mathrm{mHz}$ \\
\hline
\end{tabular}

noise of the seismometer, derive the aLIGO platform noise, and estimate the angular motion of the aLIGO test masses. We also compare our results with the performance of the currently installed T240 seismometers.

Fig. 6 shows the design of the fused silica suspension and Table 1 summarises the key parameters of the design. The customised commercially available fused silica mass [35] is circular in shape with the mass concentrated at the edges, and the three arms are designed to be as minimal as possible to reduce mass near the centre, therefore, maximising the moment of inertia for this configuration. The vertical and horizontal position of the centre of mass will be tuneable via small masses.

\subsection{Low frequency drifts}

Ideally, the angular modes of the suspended inertial mass should be as low as possible to diagonalise the tilt and translational signals of the seismometer. However, soft suspensions tend to drift at low frequencies due to stress relaxations in the fibre [36] and temperature 
gradients along the suspended mass. Strong drifts have the potential to move the system out of its interferometric sensing range. Experiments with torsion balances conducted in Washington [37], Paris, and Birmingham [38], and at the LISA facilities in Florida [39] and Trento [40] show that the fused silica suspensions drift less than the tungsten ones and that softer RZ eigenmodes lead to larger drifts.

We propose to stiffen RZ by making the fibre cross-section non-uniform: its radius will be $r=120 \mu \mathrm{m}$ near the fibre ends and $r=500 \mu \mathrm{m}$ near the centre of the fibre. The RZ eigen mode is then given by the equation

$$
f_{\mathrm{RZ}}=\frac{1}{2 \pi} \sqrt{\frac{E_{t} J}{L_{\mathrm{eff}} I_{\mathrm{RZ}}}},
$$

where $E_{t}=30 \mathrm{GPa}$ is the modulus of torsion of fused silica, $J=\pi r^{4} / 2$ is the second moment of area along the axis of the cylindrical fibre, $L_{\text {eff }}$ is the length of the fibre section with radius $r=120 \mu \mathrm{m}$. To stiffen RZ, the effective fibre length, $L_{\text {eff }}$, must satisfy the following condition

$$
2 \Delta=2 \sqrt{\frac{E I_{a}}{m g}} \ll L_{\mathrm{eff}} \ll L
$$

where $\Delta=600 \mu \mathrm{m}$ is the bending length of the fibre, $L=40 \mathrm{~cm}$ is the fibre length, and a factor of 2 accounts for two bending locations of the fibre. We choose $L_{\text {eff }}=1 \mathrm{~cm}$ to satisfy Eq. (8) and get $f_{\mathrm{RZ}}=31 \mathrm{mHz}$. We expect the drift rate of less than $1 \mu \mathrm{m} /$ week due to stress relaxation in the fibre.

Apart from the fibre unwinding, we also consider thermal gradients across the inertial mass. The gradients make different parts of the mass expand non-uniformly and cause the suspension to lose its balance. We estimate the angular deviation in RX and RY according to the equation

$$
\theta_{\mathrm{rms}} \approx \frac{g \alpha \Delta T_{\mathrm{rms}}}{2 R \omega_{\mathrm{RX}, \mathrm{RY}}^{2}}=1.6 \times \frac{\Delta T_{\mathrm{rms}}}{20 \mathrm{mK}}\left(\frac{50 \mathrm{mHz}}{f_{\mathrm{RX}, \mathrm{RY}}}\right)^{2} \mu \mathrm{rad},
$$

where $\alpha=4 \times 10^{-7} \mathrm{~K}^{-1}$ is the thermal expansion coefficient of fused silica [35], and $\Delta T_{\text {rms }}$ are thermal gradients along the inertial mass. We conclude that the thermal gradients are not significant for the proposed fused silica design.

\subsection{Projected motion of the aLIGO platforms}

The sensitivity of the inertial mass position readout is limited by the own noise of the compact interferometer, thermal noise of the suspension, and the actuation noise. The readout noise will be the dominant noise of the seismometer. We set the requirement of

$$
S_{\text {ifo }}=\left(3 \times 10^{-13}\right)^{2}+\left(\frac{10^{-13}}{f}\right)^{2} \frac{\mathrm{m}^{2}}{\mathrm{~Hz}}
$$

for the local readout of the mass position in the frequency band from $1 \mathrm{mHz}$ up to $10 \mathrm{~Hz}$. This level of sensitivity has been already achieved by the custom compact interferometers [31, 41]. 


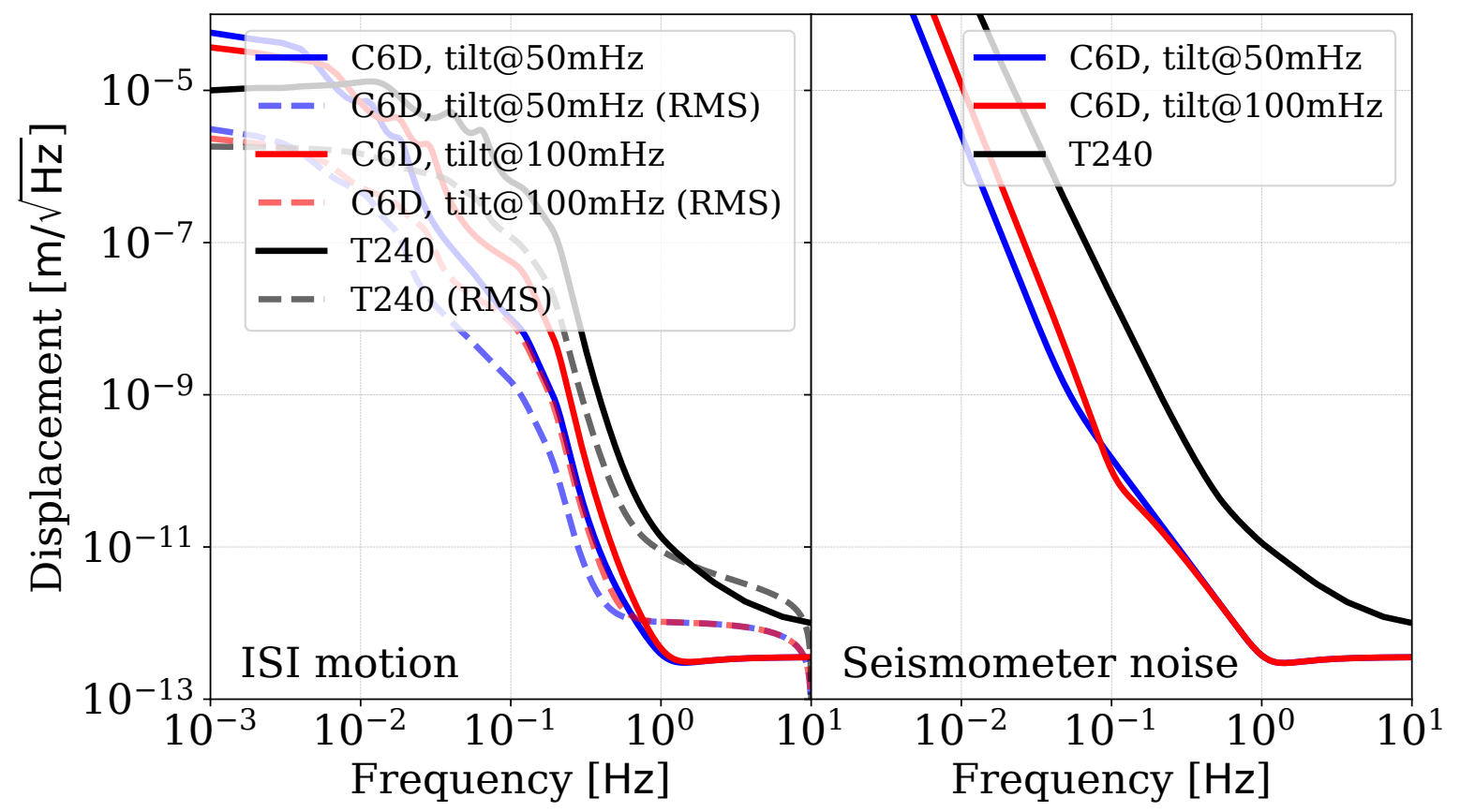

Figure 7. Estimated motion of the aLIGO platforms with the $6 \mathrm{D}$ seismometers (left). The right panel shows the comparison between the own noises of the $6 \mathrm{D}$ seismometers with the tilt eigenmode at $50 \mathrm{mHz}$ and $100 \mathrm{mHz}$, and with the own noise of the T240 seismometers.

Local readout noise couples to the estimated horizontal motion of the platform, $\hat{x}$. According to the dynamics of the system [19], the readout noise coupling to $\hat{x}$ grows as $1 / f^{2}$ below the longitudinal resonances, $f_{X, Y}$, and as $1 / f^{4}$ below the tilt resonances, $f_{\mathrm{RX}, \mathrm{RY}}$ as shown in Fig. 7. The $1 / f^{2}$ and $1 / f^{4}$ couplings are similar to the current noise scaling in the aLIGO detectors. For the T240 seismometers, the $1 / f^{2}$ slope starts from $1 \mathrm{~Hz}$ and the $1 / f^{4}$ starts from the effective tilt resonance given by the equation

$$
f_{\mathrm{RX}, \mathrm{RY}}^{\mathrm{T} 240} \approx \frac{1}{2 \pi} \sqrt{\frac{2 g}{\Delta L}}=0.7 \mathrm{~Hz} .
$$

Our calculations of the suspension thermal noise of the inertial mass show that it becomes insignificant for the quality factors of the tilt mode $Q_{t}>2 \times 10^{4}$. Since the aLIGO suspensions achieve loss angles of the suspension fibres below $10^{-6}$ [42], the required quality factors can be achieved with the proposed fused silica suspension. We propose to damp the high quality resonances with magnet-coil actuators similar to the aLIGO suspensions [43]. The damping will allow us to reduce the quality factors of the suspensions down to $\sim 10$ and simplify the diagonalisation of the readout signals according to Eq. (5). Since we need to make the actuator noise insignificant, the actuator range will be $10 \mu \mathrm{m}$ and will not be able to correct any strong low-frequency drifts. However, as discussed in Sec. 3.1, the fused silica design of the suspension will help avoid strong drifts.

The total noise of the seismometers, shown in Fig. 7, becomes larger than the ground motion below $\approx 30 \mathrm{mHz}$. In order to avoid large motions of the aLIGO platforms below $10 \mathrm{mHz}$, aLIGO utilises a blended control scheme $[8,28]$. The aLIGO platforms follow the 


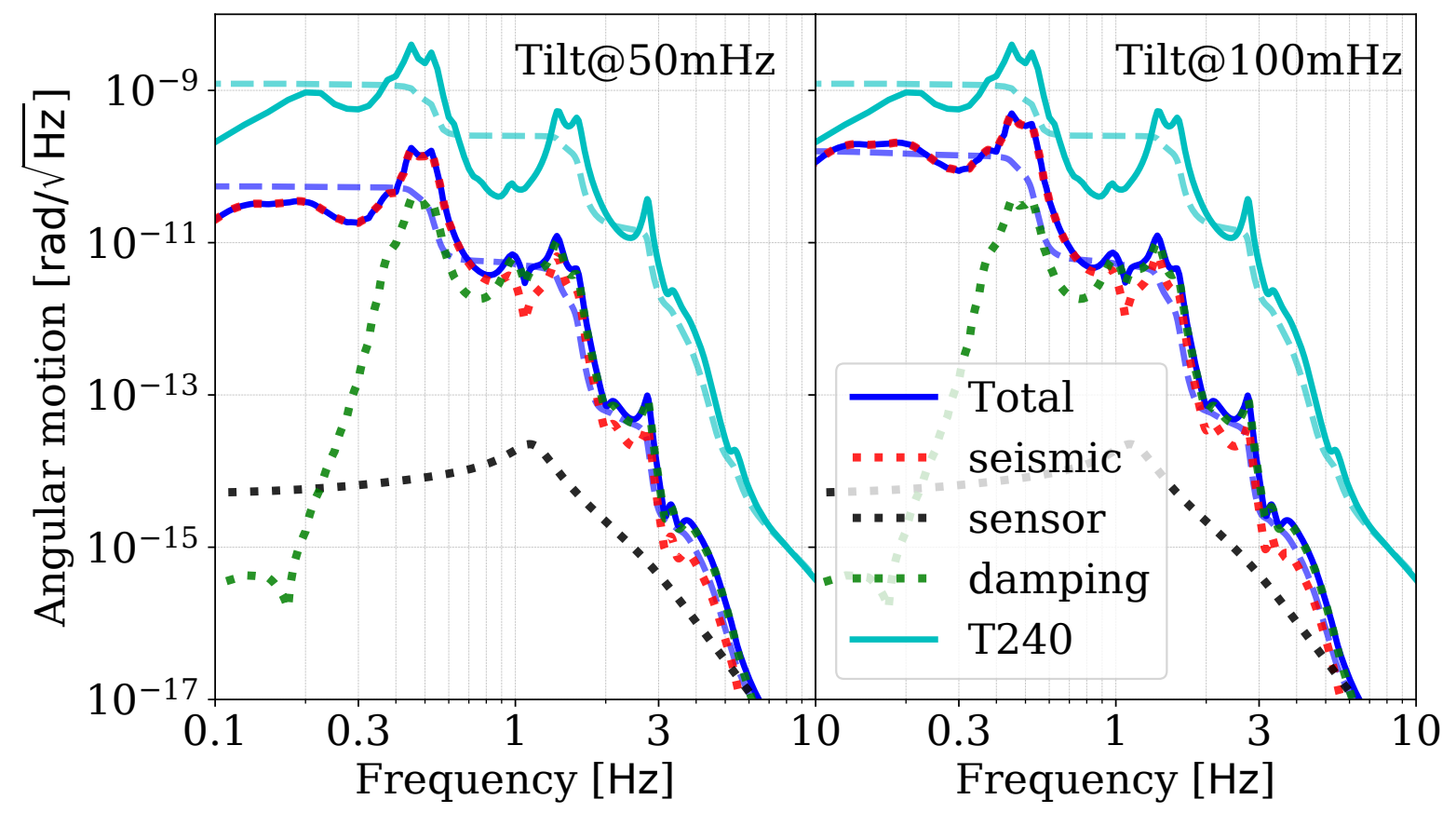

Figure 8. Comparison of the estimated pitch motion of the aLIGO test masses when the aLIGO platforms are stabilised with the proposed $6 \mathrm{D}$ seismometers with a tilt eigenmode of $50 \mathrm{mHz}$ (left panel), $100 \mathrm{mHz}$ (right panel) and the T240 seismometers.

signals from the inertial sensors only above the blending frequency. At lower frequencies, the aLIGO platforms follow the signals from the positions sensors that measure the relative motion between the aLIGO platforms and the ground. A lower blending frequency provides better seismic isolation above $100 \mathrm{mHz}$ but couples more inertial sensor noise to lower frequencies. In the aLIGO detectors, where T240 seismometers are currently deployed, the blending frequency is set at $45 \mathrm{mHz}$. The proposed 6D seismometer has the potential to lower the blending frequency by a factor of $2-3$ without increasing the RMS motion of the platform as shown in Fig. 7. In this study, we find that a $6 \mathrm{D}$ seismometer with a tilt mode at $50 \mathrm{mHz}$ $(100 \mathrm{mHz})$ can reduce the blending frequency down to $14 \mathrm{mHz}(22 \mathrm{mHz})$.

\subsection{Projected motion of the aLIGO test masses}

The key noise source of the aLIGO detectors at low frequencies (below $30 \mathrm{~Hz}$ ) is the angular control noise. The motion of the aLIGO platforms causes angular motion of the test masses. The motion is then actively stabilised by wavefront sensors [44, 45]. The control loops keep the relative motion of the aLIGO test masses below $\sim 1 \mathrm{nrad}$, but couple the wavefront sensor sensing noise to the aLIGO GW channel [46]. The bandwidth of the angular control loops is currently set to be $3 \mathrm{~Hz}$ [12]. Since the $6 \mathrm{D}$ seismometer has the potential to improve the motion of the aLIGO platforms as shown in Fig. 7, the angular controls bandwidth can be reduced by a factor of 3 and remove coupling of the aLIGO angular controls to its sensitivity band above $10 \mathrm{~Hz}$ [10].

In this section, we estimate the angular motion of the aLIGO test masses with the 
6D seismometer. The dominant angular motion of the aLIGO test masses comes from the longitudinal motion of the aLIGO platforms [10]. We propagate the platform motion through the transfer functions of the aLIGO quadruple suspensions and compute the motion of the test masses. We find that we can achieve residual relative motion of the aLIGO test masses below $1 \mathrm{nrad}$ with a control bandwidth of $1 \mathrm{~Hz}$ and a typical wavefront sensor noise of $5 \times 10^{-15} \mathrm{rad} / \sqrt{\mathrm{Hz}}$ when the tilt mode of the seismometer inertial mass is either $50 \mathrm{mHz}$ or $100 \mathrm{mHz}$. The results of mirror angular motion are shown in Fig. 8. The damping noise comes from the local sensors that damp the high quality factors of the aLIGO suspensions. In computing the damping noise for Fig. 8, we assume that the aLIGO suspensions are equipped with the same interferometeric positions sensors as the 6D seismometers.

We find that both configurations of the $6 \mathrm{D}$ seismometer from Table 1 have the potential to solve the problem of the aLIGO angular control coupling to the $\mathrm{GW}$ channel below $20 \mathrm{~Hz}$. The $100 \mathrm{mHz}$ configuration is easier to implement compared to the $50 \mathrm{mHz}$ one since center-ofmass tuning $\Delta z$ can be less precise. However, the $50 \mathrm{mHz}$ version provides significantly better inertial isolation in the earthquake band between $30 \mathrm{mHz}$ and $300 \mathrm{mHz}$, and has the potential to keep the aLIGO detectors operational during small and medium earthquakes (local RMS velocity of the ground below $\sim 1 \mu \mathrm{m} / \mathrm{s}$ ) and further improve the aLIGO duty cycle.

\section{Conclusion}

Novel inertial sensors can enhance the sensitivity of the aLIGO detectors below $30 \mathrm{~Hz}$, improve their duty cycle, and open a pathway towards increasing the beam size in the aLIGO arms. In this paper, we presented experimental results from our studies of the compact 6D seismometer with an interferometric readout. We conclude that the seismometer has the potential to improve aLIGO's seismic isolation. Measurement of the horizontal and tilt modes of the metallic prototype resulted in validation of the decoupling capabilities of the readout scheme though decoupling around the horizontal eigen mode requires further investigations. We found that lower tilt eigenmodes of the suspended mass make the balancing procedure complicated due to plastic deformations in the wires.

Using the lessons learned from the metal prototype, we proposed and simulated the performance of a fused silica compact $6 \mathrm{D}$ seismometer with the tilt modes at $50 \mathrm{mHz}$ and $100 \mathrm{mHz}$. We found that both configurations should have low angular drift rates due to the mechanical and thermal properties of fused silica. The seismometers are capable of improving the current aLIGO low frequency noise but the $50 \mathrm{mHz}$ design also provides strong suppression of the the ground motion in the earthquake band. Experimental investigations of the compact fused silica 6D seismometer will be our next step.

\section{Acknowledgements}

We thank Hang Yu, members of the LIGO SWG group and our collaborators on 6D seismometers from VU, Amsterdam for useful discussions. The authors acknowledge the support of the Institute for Gravitational Wave Astronomy at the University of Birmingham, 
STFC 2018 Equipment Call ST/S002154/1, STFC 'Astrophysics at the University of Birmingham' grant ST/S000305/1. A.S.U. and J.S. are supported by STFC studentships 2117289 and 2116965. H. M. is supported by UK STFC Ernest Rutherford Fellowship ST/M005844/11.

\section{Bibliography}

[1] J Aasi, B P Abbott, R Abbott, T Abbott, M R Abernathy, K Ackley, C Adams, T Adams, P Addesso, and et al. Advanced ligo. Classical and Quantum Gravity, 32(7):074001, Mar 2015.

[2] F. Acernese, M. Agathos, K. Agatsuma, D. Aisa, N. Allemandou, A. Allocca, J. Amarni, P. Astone, G. Balestri, G. Ballardin, F. Barone, J. P. Baronick, M. Barsuglia, A. Basti, F. Basti, Th. S. Bauer, V. Bavigadda, M. Bejger, M. G. Beker, C. Belczynski, D. Bersanetti, A. Bertolini, M. Bitossi, M. A. Bizouard, S. Bloemen, M. Blom, M. Boer, G. Bogaert, D. Bondi, F. Bondu, L. Bonelli, R. Bonnand, V. Boschi, L. Bosi, T. Bouedo, C. Bradaschia, M. Branchesi, T. Briant, A. Brillet, V. Brisson, T. Bulik, H. J. Bulten, D. Buskulic, C. Buy, G. Cagnoli, E. Calloni, C. Campeggi, B. Canuel, F. Carbognani, F. Cavalier, R. Cavalieri, G. Cella, E. Cesarini, E. Chassande-Mottin, A. Chincarini, A. Chiummo, S. Chua, F. Cleva, E. Coccia, P. F. Cohadon, A. Colla, M. Colombini, A. Conte, J. P. Coulon, E. Cuoco, A. Dalmaz, S. D’Antonio, V. Dattilo, M. Davier, R. Day, G. Debreczeni, J. Degallaix, S. Deléglise, W. Del Pozzo, H. Dereli, R. De Rosa, L. Di Fiore, A. Di Lieto, A. Di Virgilio, M. Doets, V. Dolique, M. Drago, M. Ducrot, G. Endrőczi, V. Fafone, S. Farinon, I. Ferrante, F. Ferrini, F. Fidecaro, I. Fiori, R. Flaminio, J. D. Fournier, S. Franco, S. Frasca, F. Frasconi, L. Gammaitoni, F. Garufi, M. Gaspard, A. Gatto, G. Gemme, B. Gendre, E. Genin, A. Gennai, S. Ghosh, L. Giacobone, A. Giazotto, R. Gouaty, M. Granata, G. Greco, P. Groot, G. M. Guidi, J. Harms, A. Heidmann, H. Heitmann, P. Hello, G. Hemming, E. Hennes, D. Hofman, P. Jaranowski, R. J. G. Jonker, M. Kasprzack, F. Kéfélian, I. Kowalska, M. Kraan, A. Królak, A. Kutynia, C. Lazzaro, M. Leonardi, N. Leroy, N. Letendre, T. G. F. Li, B. Lieunard, M. Lorenzini, V. Loriette, G. Losurdo, C. Magazzù, E. Majorana, I. Maksimovic, V. Malvezzi, N. Man, V. Mangano, M. Mantovani, F. Marchesoni, F. Marion, J. Marque, F. Martelli, L. Martellini, A. Masserot, D. Meacher, J. Meidam, F. Mezzani, C. Michel, L. Milano, Y. Minenkov, A. Moggi, M. Mohan, M. Montani, N. Morgado, B. Mours, F. Mul, M. F. Nagy, I. Nardecchia, L. Naticchioni, G. Nelemans, I. Neri, M. Neri, F. Nocera, E. Pacaud, C. Palomba, F. Paoletti, A. Paoli, A. Pasqualetti, R. Passaquieti, D. Passuello, M. Perciballi, S. Petit, M. Pichot, F. Piergiovanni, G. Pillant, A Piluso, L. Pinard, R. Poggiani, M. Prijatelj, G. A. Prodi, M. Punturo, P. Puppo, D. S. Rabeling, I. Rácz, P. Rapagnani, M. Razzano, V. Re, T. Regimbau, F. Ricci, F. Robinet, A. Rocchi, L. Rolland, R. Romano, D. Rosińska, P. Ruggi, E. Saracco, B. Sassolas, F. Schimmel, D. Sentenac, V. Sequino, S. Shah, K. Siellez, N. Straniero, B. Swinkels, M. Tacca, M. Tonelli, F. Travasso, M. Turconi, G. Vajente, N. van Bakel, M. van Beuzekom, J. F. J. van den Brand, C. Van Den Broeck, M. V. van der Sluys, J. van Heijningen, M. Vasúth, G. Vedovato, J. Veitch, D. Verkindt, F. Vetrano, A. Viceré, J. Y. Vinet, G. Visser, H. Vocca, R. Ward, M. Was, L. W. Wei, M. Yvert, A. Zadrożny, and J. P. Zendri. Advanced virgo: a 2nd generation interferometric gravitational wave detector. Class. Quantum Grav. 32 (2015) 024001, August 2014.

[3] Benjamin P Abbott, Richard Abbott, TD Abbott, MR Abernathy, Fausto Acernese, Kendall Ackley, Carl Adams, Thomas Adams, Paolo Addesso, RX Adhikari, et al. Observation of gravitational waves from a binary black hole merger. Physical review letters, 116(6):061102, 2016.

[4] The LIGO Scientific Collaboration and the Virgo Collaboration. Gw151226: Observation of gravitational waves from a 22-solar-mass binary black hole coalescence. Phys. Rev. Lett. 116, 241103 (2016), June 2016.

[5] The LIGO Scientific Collaboration, the Virgo Collaboration, B. P. Abbott, R. Abbott, T. D. Abbott, F. Acernese, K. Ackley, C. Adams, T. Adams, P. Addesso, R. X. Adhikari, V. B. Adya, C. Affeldt, M. Afrough, B. Agarwal, M. Agathos, K. Agatsuma, N. Aggarwal, O. D. Aguiar, L. Aiello, A. Ain, P. Ajith, B. Allen, G. Allen, A. Allocca, P. A. Altin, A. Amato, A. Ananyeva, S. B. Anderson, W. G. 
Anderson, S. V. Angelova, S. Antier, S. Appert, K. Arai, M. C. Araya, J. S. Areeda, N. Arnaud, K. G. Arun, S. Ascenzi, G. Ashton, M. Ast, S. M. Aston, P. Astone, D. V. Atallah, P. Aufmuth, C. Aulbert, K. AultONeal, C. Austin, A. Avila-Alvarez, S. Babak, P. Bacon, M. K. M. Bader, S. Bae, P. T. Baker, F. Baldaccini, G. Ballardin, S. W. Ballmer, S. Banagiri, J. C. Barayoga, S. E. Barclay, B. C. Barish, D. Barker, K. Barkett, F. Barone, B. Barr, L. Barsotti, M. Barsuglia, D. Barta, S. D. Barthelmy, J. Bartlett, I. Bartos, R. Bassiri, A. Basti, J. C. Batch, M. Bawaj, J. C. Bayley, M. Bazzan, B. B'ecsy, C. Beer, M. Bejger, I. Belahcene, A. S. Bell, B. K. Berger, G. Bergmann, J. J. Bero, C. P. L. Berry, D. Bersanetti, A. Bertolini, J. Betzwieser, S. Bhagwat, R. Bhandare, I. A. Bilenko, G. Billingsley, C. R. Billman, J. Birch, R. Birney, O. Birnholtz, S. Biscans, S. Biscoveanu, A. Bisht, M. Bitossi, C. Biwer, M. A. Bizouard, J. K. Blackburn, J. Blackman, C. D. Blair, D. G. Blair, R. M. Blair, S. Bloemen, O. Bock, N. Bode, M. Boer, G. Bogaert, A. Bohe, F. Bondu, E. Bonilla, R. Bonnand, B. A. Boom, R. Bork, V. Boschi, S. Bose, K. Bossie, Y. Bouffanais, A. Bozzi, C. Bradaschia, P. R. Brady, M. Branchesi, J. E. Brau, T. Briant, A. Brillet, M. Brinkmann, V. Brisson, P. Brockill, J. E. Broida, A. F. Brooks, D. A. Brown, D. D. Brown, S. Brunett, C. C. Buchanan, A. Buikema, T. Bulik, H. J. Bulten, A. Buonanno, D. Buskulic, C. Buy, R. L. Byer, M. Cabero, L. Cadonati, G. Cagnoli, C. Cahillane, J. Calder'on Bustillo, T. A. Callister, E. Calloni, J. B. Camp, M. Canepa, P. Canizares, K. C. Cannon, H. Cao, J. Cao, C. D. Capano, E. Capocasa, F. Carbognani, S. Caride, M. F. Carney, J. Casanueva Diaz, C. Casentini, S. Caudill, M. Cavagli‘a, F. Cavalier, R. Cavalieri, G. Cella, C. B. Cepeda, P. Cerd'a-Dur'an, G. Cerretani, E. Cesarini, S. J. Chamberlin, M. Chan, S. Chao, P. Charlton, E. Chase, E. Chassande-Mottin, D. Chatterjee, K. Chatziioannou, B. D. Cheeseboro, H. Y. Chen, X. Chen, Y. Chen, H. P. Cheng, H. Chia, A. Chincarini, A. Chiummo, T. Chmiel, H. S. Cho, M. Cho, J. H. Chow, N. Christensen, Q. Chu, A. J. K. Chua, S. Chua, A. K. W. Chung, S. Chung, G. Ciani, R. Ciolfi, C. E. Cirelli, A. Cirone, F. Clara, J. A. Clark, P. Clearwater, F. Cleva, C. Cocchieri, E. Coccia, P. F. Cohadon, D. Cohen, A. Colla, C. G. Collette, L. R. Cominsky, M. Constancio Jr., L. Conti, S. J. Cooper, P. Corban, T. R. Corbitt, I. Cordero-Carri'on, K. R. Corley, N. Cornish, A. Corsi, S. Cortese, C. A. Costa, M. W. Coughlin, S. B. Coughlin, J. P. Coulon, S. T. Countryman, P. Couvares, P. B. Covas, E. E. Cowan, D. M. Coward, M. J. Cowart, D. C. Coyne, R. Coyne, J. D. E. Creighton, T. D. Creighton, J. Cripe, S. G. Crowder, T. J. Cullen, A. Cumming, L. Cunningham, E. Cuoco, T. Dal Canton, G. D'alya, S. L. Danilishin, S. D’Antonio, K. Danzmann, A. Dasgupta, C. F. Da Silva Costa, V. Dattilo, I. Dave, M. Davier, D. Davis, E. J. Daw, B. Day, S. De, D. DeBra, J. Degallaix, M. De Laurentis, S. Del'eglise, W. Del Pozzo, N. Demos, T. Denker, T. Dent, R. De Pietri, V. Dergachev, R. De Rosa, R. T. DeRosa, C. De Rossi, R. DeSalvo, O. de Varona, J. Devenson, S. Dhurandhar, M. C. D'iaz, L. Di Fiore, M. Di Giovanni, T. Di Girolamo, A. Di Lieto, S. Di Pace, I. Di Palma, F. Di Renzo, Z. Doctor, V. Dolique, F. Donovan, K. L. Dooley, S. Doravari, I. Dorrington, R. Douglas, M. Dovale 'Alvarez, T. P. Downes, M. Drago, C. Dreissigacker, J. C. Driggers, Z. Du, M. Ducrot, P. Dupej, S. E. Dwyer, T. B. Edo, M. C. Edwards, A. Effler, H. B. Eggenstein, P. Ehrens, J. Eichholz, S. S. Eikenberry, R. A. Eisenstein, R. C. Essick, D. Estevez, Z. B. Etienne, T. Etzel, M. Evans, T. M. Evans, M. Factourovich, V. Fafone, H. Fair, S. Fairhurst, X. Fan, S. Farinon, B. Farr, W. M. Farr, E. J. Fauchon-Jones, M. Favata, M. Fays, C. Fee, H. Fehrmann, J. Feicht, M. M. Fejer, A. Fernandez-Galiana, I. Ferrante, E. C. Ferreira, F. Ferrini, F. Fidecaro, D. Finstad, I. Fiori, D. Fiorucci, M. Fishbach, R. P. Fisher, M. Fitz-Axen, R. Flaminio, M. Fletcher, H. Fong, J. A. Font, P. W. F. Forsyth, S. S. Forsyth, J. D. Fournier, S. Frasca, F. Frasconi, Z. Frei, A. Freise, R. Frey, V. Frey, E. M. Fries, P. Fritschel, V. V. Frolov, P. Fulda, M. Fyffe, H. Gabbard, B. U. Gadre, S. M. Gaebel, J. R. Gair, L. Gammaitoni, M. R. Ganija, S. G. Gaonkar, C. GarciaQuiros, F. Garufi, B. Gateley, S. Gaudio, G. Gaur, V. Gayathri, N. Gehrels, G. Gemme, E. Genin, A. Gennai, D. George, J. George, L. Gergely, V. Germain, S. Ghonge, Abhirup Ghosh, Archisman Ghosh, S. Ghosh, J. A. Giaime, K. D. Giardina, A. Giazotto, K. Gill, L. Glover, E. Goetz, R. Goetz, S. Gomes, B. Goncharov, G. Gonz' alez, J. M. Gonzalez Castro, A. Gopakumar, M. L. Gorodetsky, S. E. Gossan, M. Gosselin, R. Gouaty, A. Grado, C. Graef, M. Granata, A. Grant, S. Gras, C. Gray, G. Greco, A. C. Green, E. M. Gretarsson, P. Groot, H. Grote, S. Grunewald, P. Gruning, G. M. Guidi, X. Guo, A. Gupta, M. K. Gupta, K. E. Gushwa, E. K. Gustafson, R. Gustafson, O. Halim, B. R. Hall, E. D. Hall, E. Z. Hamilton, G. Hammond, M. Haney, M. M. Hanke, J. Hanks, C. Hanna, M. D. Hannam, 
O. A. Hannuksela, J. Hanson, T. Hardwick, J. Harms, G. M. Harry, I. W. Harry, M. J. Hart, C. J. Haster, K. Haughian, J. Healy, A. Heidmann, M. C. Heintze, H. Heitmann, P. Hello, G. Hemming, M. Hendry, I. S. Heng, J. Hennig, A. W. Heptonstall, M. Heurs, S. Hild, T. Hinderer, D. Hoak, D. Hofman, K. Holt, D. E. Holz, P. Hopkins, C. Horst, J. Hough, E. A. Houston, E. J. Howell, Y. M. Hu, E. A. Huerta, D. Huet, B. Hughey, S. Husa, S. H. Huttner, T. Huynh-Dinh, N. Indik, R. Inta, G. Intini, H. N. Isa, J. M. Isac, M. Isi, B. R. Iyer, K. Izumi, T. Jacqmin, K. Jani, P. Jaranowski, S. Jawahar, F. Jim'enez-Forteza, W. W. Johnson, N. K. Johnson-McDaniel, D. I. Jones, R. Jones, R. J. G. Jonker, L. Ju, J. Junker, C. V. Kalaghatgi, V. Kalogera, B. Kamai, S. Kandhasamy, G. Kang, J. B. Kanner, S. J. Kapadia, S. Karki, K. S. Karvinen, M. Kasprzack, M. Katolik, E. Katsavounidis, W. Katzman, S. Kaufer, K. Kawabe, F. K'ef'elian, D. Keitel, A. J. Kemball, R. Kennedy, C. Kent, J. S. Key, F. Y. Khalili, I. Khan, S. Khan, Z. Khan, E. A. Khazanov, N. Kijbunchoo, Chunglee Kim, J. C. Kim, K. Kim, W. Kim, W. S. Kim, Y. M. Kim, S. J. Kimbrell, E. J. King, P. J. King, M. Kinley-Hanlon, R. Kirchhoff, J. S. Kissel, L. Kleybolte, S. Klimenko, T. D. Knowles, P. Koch, S. M. Koehlenbeck, S. Koley, V. Kondrashov, A. Kontos, M. Korobko, W. Z. Korth, I. Kowalska, D. B. Kozak, C. Kr"amer, V. Kringel, B. Krishnan, A. Kr'olak, G. Kuehn, P. Kumar, R. Kumar, S. Kumar, L. Kuo, A. Kutynia, S. Kwang, B. D. Lackey, K. H. Lai, M. Landry, R. N. Lang, J. Lange, B. Lantz, R. K. Lanza, A. Lartaux-Vollard, P. D. Lasky, M. Laxen, A. Lazzarini, C. Lazzaro, P. Leaci, S. Leavey, C. H. Lee, H. K. Lee, H. M. Lee, H. W. Lee, K. Lee, J. Lehmann, A. Lenon, M. Leonardi, N. Leroy, N. Letendre, Y. Levin, T. G. F. Li, S. D. Linker, T. B. Littenberg, J. Liu, R. K. L. Lo, N. A. Lockerbie, L. T. London, J. E. Lord, M. Lorenzini, V. Loriette, M. Lormand, G. Losurdo, J. D. Lough, C. O. Lousto, G. Lovelace, H. L"uck, D. Lumaca, A. P. Lundgren, R. Lynch, Y. Ma, R. Macas, S. Macfoy, B. Machenschalk, M. MacInnis, D. M. Macleod, I. Magaña Hernandez, F. Magaña-Sandoval, L. Magaña Zertuche, R. M. Magee, E. Majorana, I. Maksimovic, N. Man, V. Mandic, V. Mangano, G. L. Mansell, M. Manske, M. Mantovani, F. Marchesoni, F. Marion, S. M'arka, Z. M'arka, C. Markakis, A. S. Markosyan, A. Markowitz, E. Maros, A. Marquina, P. Marsh, F. Martelli, L. Martellini, I. W. Martin, R. M. Martin, D. V. Martynov, K. Mason, E. Massera, A. Masserot, T. J. Massinger, M. Masso-Reid, S. Mastrogiovanni, A. Matas, F. Matichard, L. Matone, N. Mavalvala, N. Mazumder, R. McCarthy, D. E. McClelland, S. McCormick, L. McCuller, S. C. McGuire, G. McIntyre, J. McIver, D. J. McManus, L. McNeill, T. McRae, S. T. McWilliams, D. Meacher, G. D. Meadors, M. Mehmet, J. Meidam, E. Mejuto-Villa, A. Melatos, G. Mendell, R. A. Mercer, E. L. Merilh, M. Merzougui, S. Meshkov, C. Messenger, C. Messick, R. Metzdorff, P. M. Meyers, H. Miao, C. Michel, H. Middleton, E. E. Mikhailov, L. Milano, A. L. Miller, B. B. Miller, J. Miller, M. Millhouse, M. C. Milovich-Goff, O. Minazzoli, Y. Minenkov, J. Ming, C. Mishra, S. Mitra, V. P. Mitrofanov, G. Mitselmakher, R. Mittleman, D. Moffa, A. Moggi, K. Mogushi, M. Mohan, S. R. P. Mohapatra, M. Montani, C. J. Moore, D. Moraru, G. Moreno, S. Morisaki, S. R. Morriss, B. Mours, C. M. Mow-Lowry, G. Mueller, A. W. Muir, Arunava Mukherjee, D. Mukherjee, S. Mukherjee, N. Mukund, A. Mullavey, J. Munch, E. A. Muñiz, M. Muratore, P. G. Murray, K. Napier, I. Nardecchia, L. Naticchioni, R. K. Nayak, J. Neilson, G. Nelemans, T. J. N. Nelson, M. Nery, A. Neunzert, L. Nevin, J. M. Newport, G. Newton, K. K. Y. Ng, T. T. Nguyen, D. Nichols, A. B. Nielsen, S. Nissanke, A. Nitz, A. Noack, F. Nocera, D. Nolting, C. North, L. K. Nuttall, J. Oberling, G. D. O’Dea, G. H. Ogin, J. J. Oh, S. H. Oh, F. Ohme, M. A. Okada, M. Oliver, P. Oppermann, Richard J. Oram, B. O'Reilly, R. Ormiston, L. F. Ortega, R. O'Shaughnessy, S. Ossokine, D. J. Ottaway, H. Overmier, B. J. Owen, A. E. Pace, J. Page, M. A. Page, A. Pai, S. A. Pai, J. R. Palamos, O. Palashov, C. Palomba, A. Pal-Singh, Howard Pan, Huang-Wei Pan, B. Pang, P. T. H. Pang, C. Pankow, F. Pannarale, B. C. Pant, F. Paoletti, A. Paoli, M. A. Papa, A. Parida, W. Parker, D. Pascucci, A. Pasqualetti, R. Passaquieti, D. Passuello, M. Patil, B. Patricelli, B. L. Pearlstone, M. Pedraza, R. Pedurand, L. Pekowsky, A. Pele, S. Penn, C. J. Perez, A. Perreca, L. M. Perri, H. P. Pfeiffer, M. Phelps, O. J. Piccinni, M. Pichot, F. Piergiovanni, V. Pierro, G. Pillant, L. Pinard, I. M. Pinto, M. Pirello, M. Pitkin, M. Poe, R. Poggiani, P. Popolizio, E. K. Porter, A. Post, J. Powell, J. Prasad, J. W. W. Pratt, G. Pratten, V. Predoi, T. Prestegard, M. Prijatelj, M. Principe, S. Privitera, R. Prix, G. A. Prodi, L. G. Prokhorov, O. Puncken, M. Punturo, P. Puppo, M. P"urrer, H. Qi, V. Quetschke, E. A. Quintero, R. Quitzow-James, F. J. Raab, D. S. Rabeling, H. Radkins, P. Raffai, S. Raja, C. Rajan, B. Rajbhandari, M. Rakhmanov, K. E. Ramirez, A. Ramos-Buades, P. Rapagnani, 
V. Raymond, M. Razzano, J. Read, T. Regimbau, L. Rei, S. Reid, D. H. Reitze, W. Ren, S. D. Reyes, F. Ricci, P. M. Ricker, S. Rieger, K. Riles, M. Rizzo, N. A. Robertson, R. Robie, F. Robinet, A. Rocchi, L. Rolland, J. G. Rollins, V. J. Roma, J. D. Romano, R. Romano, C. L. Romel, J. H. Romie, D. Rosi'nska, M. P. Ross, S. Rowan, A. R"udiger, P. Ruggi, G. Rutins, K. Ryan, S. Sachdev, T. Sadecki, L. Sadeghian, M. Sakellariadou, L. Salconi, M. Saleem, F. Salemi, A. Samajdar, L. Sammut, L. M. Sampson, E. J. Sanchez, L. E. Sanchez, N. Sanchis-Gual, V. Sandberg, J. R. Sanders, B. Sassolas, B. S. Sathyaprakash, P. R. Saulson, O. Sauter, R. L. Savage, A. Sawadsky, P. Schale, M. Scheel, J. Scheuer, J. Schmidt, P. Schmidt, R. Schnabel, R. M. S. Schofield, A. Sch"onbeck, E. Schreiber, D. Schuette, B. W. Schulte, B. F. Schutz, S. G. Schwalbe, J. Scott, S. M. Scott, E. Seidel, D. Sellers, A. S. Sengupta, D. Sentenac, V. Sequino, A. Sergeev, D. A. Shaddock, T. J. Shaffer, A. A. Shah, M. S. Shahriar, M. B. Shaner, L. Shao, B. Shapiro, P. Shawhan, A. Sheperd, D. H. Shoemaker, D. M. Shoemaker, K. Siellez, X. Siemens, M. Sieniawska, D. Sigg, A. D. Silva, L. P. Singer, A. Singh, A. Singhal, A. M. Sintes, B. J. J. Slagmolen, B. Smith, J. R. Smith, R. J. E. Smith, S. Somala, E. J. Son, J. A. Sonnenberg, B. Sorazu, F. Sorrentino, T. Souradeep, A. P. Spencer, A. K. Srivastava, K. Staats, A. Staley, M. Steinke, J. Steinlechner, S. Steinlechner, D. Steinmeyer, S. P. Stevenson, R. Stone, D. J. Stops, K. A. Strain, G. Stratta, S. E. Strigin, A. Strunk, R. Sturani, A. L. Stuver, T. Z. Summerscales, L. Sun, S. Sunil, J. Suresh, P. J. Sutton, B. L. Swinkels, M. J. Szczepa'nczyk, M. Tacca, S. C. Tait, C. Talbot, D. Talukder, D. B. Tanner, M. T'apai, A. Taracchini, J. D. Tasson, J. A. Taylor, R. Taylor, S. V. Tewari, T. Theeg, F. Thies, E. G. Thomas, M. Thomas, P. Thomas, K. A. Thorne, E. Thrane, S. Tiwari, V. Tiwari, K. V. Tokmakov, K. Toland, M. Tonelli, Z. Tornasi, A. Torres-Forn'e, C. I. Torrie, D. T"oyr"a, F. Travasso, G. Traylor, J. Trinastic, M. C. Tringali, L. Trozzo, K. W. Tsang, M. Tse, R. Tso, L. Tsukada, D. Tsuna, D. Tuyenbayev, K. Ueno, D. Ugolini, C. S. Unnikrishnan, A. L. Urban, S. A. Usman, H. Vahlbruch, G. Vajente, G. Valdes, M. Vallisneri, N. van Bakel, M. van Beuzekom, J. F. J. van den Brand, C. Van Den Broeck, D. C. Vander-Hyde, L. van der Schaaf, J. V. van Heijningen, A. A. van Veggel, M. Vardaro, V. Varma, S. Vass, M. Vas'uth, A. Vecchio, G. Vedovato, J. Veitch, P. J. Veitch, K. Venkateswara, G. Venugopalan, D. Verkindt, F. Vetrano, A. Vicer'e, A. D. Viets, S. Vinciguerra, D. J. Vine, J. Y. Vinet, S. Vitale, T. Vo, H. Vocca, C. Vorvick, S. P. Vyatchanin, A. R. Wade, L. E. Wade, M. Wade, R. Walet, M. Walker, L. Wallace, S. Walsh, G. Wang, H. Wang, J. Z. Wang, W. H. Wang, Y. F. Wang, R. L. Ward, J. Warner, M. Was, J. Watchi, B. Weaver, L. W. Wei, M. Weinert, A. J. Weinstein, R. Weiss, L. Wen, E. K. Wessel, P. Wessels, J. Westerweck, T. Westphal, K. Wette, J. T. Whelan, S. E. Whitcomb, B. F. Whiting, C. Whittle, D. Wilken, D. Williams, R. D. Williams, A. R. Williamson, J. L. Willis, B. Willke, M. H. Wimmer, W. Winkler, C. C. Wipf, H. Wittel, G. Woan, J. Woehler, J. Wofford, K. W. K. Wong, J. Worden, J. L. Wright, D. S. Wu, D. M. Wysocki, S. Xiao, H. Yamamoto, C. C. Yancey, L. Yang, M. J. Yap, M. Yazback, Hang Yu, Haocun Yu, M. Yvert, A. Zadro. zny, M. Zanolin, T. Zelenova, J. P. Zendri, M. Zevin, L. Zhang, M. Zhang, T. Zhang, Y. H. Zhang, C. Zhao, M. Zhou, Z. Zhou, S. J. Zhu, X. J. Zhu, A. B. Zimmerman, M. E. Zucker, and J. Zweizig. Gw170814: A three-detector observation of gravitational waves from a binary black hole coalescence. Phys. Rev. Lett. 119, 141101 (2017), September 2017.

[6] The LIGO Scientific Collaboration and The Virgo Collaboration. Gw170817: Observation of gravitational waves from a binary neutron star inspiral. Phys. Rev. Lett. 119161101 (2017), October 2017.

[7] R. Abbott et al. Gw190521: A binary black hole merger with a total mass of 150 m. Phys. Rev. Lett., 125(10):101102, 2020.

[8] F Matichard, B Lantz, R Mittleman, K Mason, J Kissel, B Abbott, S Biscans, J McIver, R Abbott, S Abbott, E Allwine, S Barnum, J Birch, C Celerier, D Clark, D Coyne, D DeBra, R DeRosa, M Evans, S Foley, P Fritschel, J A Giaime, C Gray, G Grabeel, J Hanson, C Hardham, M Hillard, W Hua, C Kucharczyk, M Landry, A Le Roux, V Lhuillier, D Macleod, M Macinnis, R Mitchell, B O’Reilly, D Ottaway, H Paris, A Pele, M Puma, H Radkins, C Ramet, M Robinson, L Ruet, P Sarin, D Shoemaker, A Stein, J Thomas, M Vargas, K Venkateswara, J Warner, and S Wen. Seismic isolation of advanced LIGO: Review of strategy, instrumentation and performance. Classical and Quantum Gravity, 32(18):185003, aug 2015.

[9] F. Matichard, B. Lantz, K. Mason, R. Mittleman, B. Abbott, S. Abbott, E. Allwine, S. Barnum, J. Birch, S. Biscans, D. Clark, D. Coyne, D. DeBra, R. DeRosa, S. Foley, P. Fritschel, J.A. Giaime, C. Gray, 
G. Grabeel, J. Hanson, M. Hillard, J. Kissel, C. Kucharczyk, A. Le Roux, V. Lhuillier, M. Macinnis, B. O’Reilly, D. Ottaway, H. Paris, M. Puma, H. Radkins, C. Ramet, M. Robinson, L. Ruet, P. Sareen, D. Shoemaker, A. Stein, J. Thomas, M. Vargas, and J. Warner. Advanced ligo two-stage twelveaxis vibration isolation and positioning platform. part 2: Experimental investigation and tests results. Precision Engineering, 40:287 - 297, 2015.

[10] Hang Yu, Denis Martynov, Salvatore Vitale, Matthew Evans, David Shoemaker, Bryan Barr, Giles Hammond, Stefan Hild, James Hough, Sabina Huttner, Sheila Rowan, Borja Sorazu, Ludovico Carbone, Andreas Freise, Conor Mow-Lowry, Katherine L. Dooley, Paul Fulda, Hartmut Grote, and Daniel Sigg. Prospects for detecting gravitational waves at $5 \mathrm{hz}$ with ground-based detectors. Phys. Rev. Lett., 120:141102, Apr 2018.

[11] Aaron Buikema et al. Sensitivity and performance of the advanced ligo detectors in the third observing run. Phys. Rev. D, 102(6):062003, 2020.

[12] D. V. Martynov, E. D. Hall, B. P. Abbott, et al. Sensitivity of the Advanced LIGO detectors at the beginning of gravitational wave astronomy. Phys. Rev. D, 93:112004, Jun 2016.

[13] A Staley, D Martynov, R Abbott, R X Adhikari, K Arai, S Ballmer, L Barsotti, A F Brooks, R T DeRosa, S Dwyer, A Effler, M Evans, P Fritschel, V V Frolov, C Gray, C J Guido, R Gustafson, M Heintze, D Hoak, K Izumi, K Kawabe, E J King, J S Kissel, K Kokeyama, M Landry, D E McClelland, J Miller, A Mullavey, B O?Reilly, J G Rollins, J R Sanders, R M S Schofield, D Sigg, B J J Slagmolen, N D SmithLefebvre, G Vajente, R L Ward, and C Wipf. Achieving resonance in the Advanced LIGO gravitationalwave interferometer. Classical and Quantum Gravity, 31(24):245010, 2014.

[14] D. Martynov. Lock Acquisition and Sensitivity Analysis of Advanced LIGO Interferometers. PhD thesis, Caltech, 2015.

[15] G. Harry, T.P. Bodiya, and R. DeSalvo. Optical Coatings and Thermal Noise in Precision Measurement. Cambridge University Press, 2012.

[16] S. Gras, H. Yu, W. Yam, D. Martynov, and M. Evans. Audio-band coating thermal noise measurement for advanced ligo with a multimode optical resonator. Phys. Rev. D, 95:022001, Jan 2017.

[17] D. V. Martynov, V. V. Frolov, S. Kandhasamy, K. Izumi, H. Miao, N. Mavalvala, E. D. Hall, R. Lanza, B. P. Abbott, R. Abbott, T. D. Abbott, C. Adams, R. X. Adhikari, S. B. Anderson, A. Ananyeva, S. Appert, K. Arai, S. M. Aston, S. W. Ballmer, D. Barker, B. Barr, L. Barsotti, J. Bartlett, I. Bartos, J. C. Batch, A. S. Bell, J. Betzwieser, G. Billingsley, J. Birch, S. Biscans, C. Biwer, C. D. Blair, R. Bork, A. F. Brooks, G. Ciani, F. Clara, S. T. Countryman, M. J. Cowart, D. C. Coyne, A. Cumming, L. Cunningham, K. Danzmann, C. F. Da Silva Costa, E. J. Daw, D. DeBra, R. T. DeRosa, R. DeSalvo, K. L. Dooley, S. Doravari, J. C. Driggers, S. E. Dwyer, A. Effler, T. Etzel, M. Evans, T. M. Evans, M. Factourovich, H. Fair, A. Fernández Galiana, R. P. Fisher, P. Fritschel, P. Fulda, M. Fyffe, J. A. Giaime, K. D. Giardina, E. Goetz, R. Goetz, S. Gras, C. Gray, H. Grote, K. E. Gushwa, E. K. Gustafson, R. Gustafson, G. Hammond, J. Hanks, J. Hanson, T. Hardwick, G. M. Harry, M. C. Heintze, A. W. Heptonstall, J. Hough, R. Jones, S. Karki, M. Kasprzack, S. Kaufer, K. Kawabe, N. Kijbunchoo, E. J. King, P. J. King, J. S. Kissel, W. Z. Korth, G. Kuehn, M. Landry, B. Lantz, N. A. Lockerbie, M. Lormand, A. P. Lundgren, M. MacInnis, D. M. Macleod, S. Márka, Z. Márka, A. S. Markosyan, E. Maros, I. W. Martin, K. Mason, T. J. Massinger, F. Matichard, R. McCarthy, D. E. McClelland, S. McCormick, G. McIntyre, J. McIver, G. Mendell, E. L. Merilh, P. M. Meyers, J. Miller, R. Mittleman, G. Moreno, G. Mueller, A. Mullavey, J. Munch, L. K. Nuttall, J. Oberling, P. Oppermann, Richard J. Oram, B. O'Reilly, D. J. Ottaway, H. Overmier, J. R. Palamos, H. R. Paris, W. Parker, A. Pele, S. Penn, M. Phelps, V. Pierro, I. Pinto, M. Principe, L. G. Prokhorov, O. Puncken, V. Quetschke, E. A. Quintero, F. J. Raab, H. Radkins, P. Raffai, S. Reid, D. H. Reitze, N. A. Robertson, J. G. Rollins, V. J. Roma, J. H. Romie, S. Rowan, K. Ryan, T. Sadecki, E. J. Sanchez, V. Sandberg, R. L. Savage, R. M. S. Schofield, D. Sellers, D. A. Shaddock, T. J. Shaffer, B. Shapiro, P. Shawhan, D. H. Shoemaker, D. Sigg, B. J. J. Slagmolen, B. Smith, J. R. Smith, B. Sorazu, A. Staley, K. A. Strain, D. B. Tanner, R. Taylor, M. Thomas, P. Thomas, K. A. Thorne, E. Thrane, C. I. Torrie, G. Traylor, G. Vajente, G. Valdes, A. A. van Veggel, A. Vecchio, P. J. Veitch, K. Venkateswara, T. Vo, C. Vorvick, M. Walker, R. L. Ward, J. Warner, B. Weaver, R. Weiss, P. Weßels, B. Willke, C. C. Wipf, J. Worden, G. Wu, H. Yamamoto, C. C. Yancey, Hang Yu, Haocun 
Yu, L. Zhang, M. E. Zucker, and J. Zweizig. Quantum correlation measurements in interferometric gravitational-wave detectors. Phys. Rev. A, 95:043831, Apr 2017.

[18] Krishna Venkateswara, Charles Hagedorn, Matthew Turner, Trevor Arp, and Jens Gundlach. A highprecision mechanical absolute-rotation sensor. The Review of scientific instruments, 85:015005, 022014.

[19] C. M. Mow-Lowry and D. Martynov. A 6d interferometric inertial isolation system. Classical and Quantum Gravity, 36(24):245006, December 2019.

[20] C. Collette, F. Nassif, J. Amar, C. Depouhon, and S.-P. Gorza. Prototype of interferometric absolute motion sensor. Sensors and Actuators, A: Physical, 224:72-77, 2015.

[21] W Z Korth, A Heptonstall, E D Hall, K Arai, E K Gustafson, and R X Adhikari. Passive, free-space heterodyne laser gyroscope. Classical and Quantum Gravity, 33(3):035004, jan 2016.

[22] Denis Martynov, Nicolas Brown, Eber Nolasco-Martinez, and Matthew Evans. Passive optical gyroscope with double homodyne readout. Opt. Lett., 44(7):1584-1587, Apr 2019.

[23] J. V. van Heijningen, A. Bertolini, and J. F. J. van den Brand. A novel interferometrically read out inertial sensor for future gravitational wave detectors. In 2018 IEEE Sensors Applications Symposium (SAS), pages 1-5, 2018.

[24] J. J. McCann, J. Winterflood, L. Ju, and C. Zhao. A multi-orientation low-frequency rotational accelerometer. Review of Scientific Instruments, 92(6):064503, 2021.

[25] Brian Lantz. Tilt-horizontal coupling for a simple inverted pendulum, 2006.

[26] B Lantz, R Schofield, B reilly, D. Clark, and Dan Debra. Review: Requirements for a ground rotation sensor to improve advanced ligo. Bulletin of The Seismological Society of America - BULL SEISMOL SOC AMER, 99, 052009.

[27] Fabrice Matichard and Matthew Evans. Review: Tilt-free low-noise seismometry. Bulletin of the Seismological Society of America, 105:497-510, 032015.

[28] F. Matichard, M. Evans, R. Mittleman, M. MacInnis, S. Biscans, K. L. Dooley, H. Sohier, A. Lauriero, H. Paris, J. Koch, P. Knothe, A. Carbajo, and C. Dufort. Modeling and experiment of the suspended seismometer concept for attenuating the contribution of tilt motion in horizontal measurements. Review of Scientific Instruments, 87:065002, 2016.

[29] Stephan Theil. Drag-free satellite control. Lasers, Clocks and Drag-Free Control, January 2008.

[30] Sercel. Seismometers: Analog seismic sensors. https://www.sercel.com/products/Pages/ seismometers. aspx. (accessed: 14.09.2021).

[31] S J Cooper, C J Collins, A C Green, D Hoyland, C C Speake, A Freise, and C M Mow-Lowry. A compact, large-range interferometer for precision measurement and inertial sensing. Classical and Quantum Gravity, 35(9):095007, mar 2018.

[32] Jennifer Watchi, Sam Cooper, Binlei Ding, Conor M. Mow-Lowry, and Christophe Collette. Contributed review: A review of compact interferometers. Review of Scientific Instruments, 89(12):121501, 2018.

[33] PASSCAL. Nanometrics trillium 240 broadband sensor. https://www. passcal. nmt. edu/content/ instrumentation/sensors/broadband-sensors/t240-bb-sensor. (accessed: 14.09.2021).

[34] AV Cumming, AS Bell, L Barsotti, MA Barton, G Cagnoli, D Cook, L Cunningham, M Evans, GD Hammond, GM Harry, et al. Design and development of the advanced ligo monolithic fused silica suspension. Classical and Quantum Gravity, 29(3):035003, 2012.

[35] Heraeus. Heraeus. https://www. heraeus.com/. (accessed: 14.09.2021).

[36] Yuri Levin. Creep events and creep noise in gravitational-wave interferometers: Basic formalism and stationary limit. Phys. Rev. D, 86:122004, Dec 2012.

[37] E. G. Adelberger, C. W. Stubbs, Blayne R. Heckel, Y. Su, H. E. Swanson, G. Smith, J. H. Gundlach, and W. F. Rogers. Testing the equivalence principle in the field of the earth: Particle physics at masses below 1-microev? Phys. Rev. D, 42:3267-3292, 1990.

[38] Terry Quinn, Harold Parks, Clive Speake, and Richard Davis. Improved determination of $g$ using two methods. Phys. Rev. Lett., 111:101102, Sep 2013.

[39] Giacomo Ciani, Andrew Chilton, Stephen Apple, Taiwo Olatunde, Michael Aitken, Guido Mueller, and John W. Conklin. A new torsion pendulum for gravitational reference sensor technology development. Review of Scientific Instruments, 88:064502, 2017. 
[40] Giuliana Russano. A torsion pendulum ground test of the lisa pathfinder free-fall mode. arXiv e-prints, page arXiv:1609.00002, August 2016.

[41] Katharina-Sophie Isleif, Gerhard Heinzel, Moritz Mehmet, and Oliver Gerberding. Compact multifringe interferometry with subpicometer precision. Phys. Rev. Applied, 12:034025, Sep 2019.

[42] A. V. Cumming, B. Sorazu, E. Daw, G. D. Hammond, J. Hough, R. Jones, I. W. Martin, S. Rowan, K. A. Strain, and D. Williams. Lowest observed surface and weld losses in fused silica fibres for gravitational wave detectors. Class. Quant. Grav., 37(19):195019, 2020.

[43] K. A. Strain and B. N. Shapiro. Damping and local control of mirror suspensions for laser interferometric gravitational wave detectors. Rev. Sci. Instrum., 83:044501, 2012.

[44] N. Mavalvala, D. Sigg, and D. Shoemaker. Experimental Test of an Alignment-Sensing Scheme for a Gravitational-Wave Interferometer. Applied optics, 37:7743-7746, November 1998.

[45] L Barsotti, M Evans, and P Fritschel. Alignment sensing and control in Advanced LIGO. Classical and Quantum Gravity, 27(8):084026, 2010.

[46] Katherine L. Dooley, Lisa Barsotti, Rana X. Adhikari, Matthew Evans, Tobin T. Fricke, Peter Fritschel, Valera Frolov, Keita Kawabe, and Nicolás Smith-Lefebvre. Angular control of optical cavities in a radiation-pressure-dominated regime: the enhanced ligo case. J. Opt. Soc. Am. A, 30(12):2618-2626, Dec 2013. 